\title{
Anaplastic Thyroid Carcinoma with Ocular Then Orbital Metastases
}

\author{
Tu Tran $^{a}$ Sanja Cypen ${ }^{b} \quad$ Maria Del Valle Estopinal ${ }^{b} \quad$ Jeremiah Tao $^{b}$ \\ ${ }^{a}$ Department of Medicine, University of California, Irvine, Irvine, CA, USA; ${ }^{b}$ Gavin Herbert \\ Eye Institute, University of California, Irvine, Irvine, CA, USA
}

\section{Keywords}

Anaplastic thyroid carcinoma $\cdot$ Choroidal metastasis $\cdot$ Orbit

\begin{abstract}
A 52-year-old Hispanic woman with a history of metastatic thyroid carcinoma presented with left eye degeneration and a choroidal mass without evidence of orbital disease on neuroimaging. She underwent enucleation for a blind, painful eye. Histopathology demonstrated choroidal metastasis of thyroid carcinoma with a well-circumscribed episcleral nodule, consistent with extraocular tumor extension. The interdisciplinary team recommended expectant management. Three months later, she developed periorbital edema and discomfort with poor ocular prosthesis fit. Neuroimaging revealed a mass in the anophthalmic socket that proved to be thyroid carcinoma. The patient subsequently underwent exenteration for pain control and local tumor burden reduction. At 12-month follow-up, there was no evidence of residual orbital disease, and she remains in remission. While the orbit involvement likely represents direct extraocular extension, a discrete orbital metastasis remains a possibility. In either scenario, this is the only known case of anaplastic thyroid carcinoma metastatic to the choroid then orbit.
\end{abstract}

\section{Introduction}

Metastasis from solid tumors comprises only a small subset (1-13\%) of orbital tumors [1-3]. Breast, prostate, and lung are the most common primary solid tumors metastasizing to the orbit [1]. There are only 22 reported cases of choroidal metastasis from thyroid carcinomas [4]. Anaplastic thyroid carcinoma (ATC) is an aggressive, regionally destructive, and 
Tran et al.: Ocular and Orbital Metastasis from Thyroid Carcinoma

fatal disease with a median survival of 5-6 months from diagnosis [5]. There has only been one reported case of ATC with ocular involvement [6]. In this report, the authors present a case of ATC arising from transformation of a papillary thyroid carcinoma (PTC) with metastasis to the choroid and subsequently the same orbit.

\section{Case Presentation}

A 52-year-old Hispanic woman with a history of serous borderline tumor of the left ovary ( $p T 1 a N x, 8$ th edition, AJJC staging manual), granulosa cell tumor in the right ovary (pT1aNx), and a thyroid mass underwent total thyroidectomy, laryngopharyngectomy, and cervical lymph node dissection at another institution prior to presenting to the authors' care. Histopathology revealed an anaplastic carcinoma within the thyroid mass with squamoid differentiation arising in association with PTC and papillary morphology in the lymph nodes ( $p T 4 a p N 1 b$ ). She was treated with systemic carboplatin, paclitaxel, pembrolizumab, and radiation to the neck.

Eight months after diagnosis of the primary thyroid tumor, she experienced subacute vision loss in the left eye. On examination by an outside provider, visual acuity in the left eye was 20/80. A large exudative retinal detachment overlying a choroidal mass was noted on fundoscopy. MRI of the brain revealed no central nervous system lesions. This presumed ocular metastasis of thyroid carcinoma was treated with 37.5 Gy fractionated external beam radiation therapy (EBRT) to the left eye and orbit with good response based on serial ophthalmic examinations.

Five months later (13 months after initial thyroid tumor diagnosis), she presented with unilateral facial droop. Neuroimaging revealed new left occipital lobe lesions, presumptive brain metastases from thyroid carcinoma, which were treated with Gamma Knife (Elektra, Stockholm, Sweden) stereotactic radiosurgery (SRS) with good response and stability of lesions on serial neuroimaging.

She subsequently developed increasing left eye pain secondary to neovascular glaucoma, treated with intravitreal bevacizumab, anterior chamber (AC) paracentesis, and intraocular pressure (IOP)-lowering eye drops. The glaucoma and eye pain progressed, becoming refractory to standard therapies.

On presentation to the authors at this time point (16 months after initial thyroid tumor diagnosis and 8 months after the identification of presumed choroidal metastasis), visual acuity diminished to light perception in the left eye, while right eye was still 20/20. Intraocular pressure was 14 and $55 \mathrm{~mm} \mathrm{Hg}$ in the right and left eye, respectively. The anterior segment examination of the left eye revealed diffuse conjunctival injection, microcystic corneal edema, and anterior chamber flare. There was extensive iris neovascularization with anterior and posterior synechiae. The right eye examination was unremarkable. B-scan ultrasound of the left eye demonstrated a choroidal mass in the posterior pole with an overlying funnel-shaped retinal detachment. MRI also demonstrated a posterior intraocular lesion in an intact eye with an area of posterior scleral enhancement, though no extraocular extension was appreciated (Fig. 1a). An AC paracentesis was performed to temporarily alleviate the pain from elevated IOP. The vision deteriorated to no light perception (NLP). As the patient now had a blind painful eye with presumed recurrence of the metastatic lesion, enucleation was recommended. Surgery was uneventful, and the eye was removed with the sclera grossly intact circumferentially. There was no notable orbital pathology on gross inspection, and a silicone sphere wrapped with autologous temporalis fascia was implanted into the socket.

Under microscopy, a dome-shaped choroidal lesion with an adjacent mushroom-shaped episcleral nodule was evident (Fig. 2a). Trabeculae and cords of neoplastic epithelioidsquamoid cells with marked nuclear pleomorphism, abundant eosinophilic cytoplasm, and 

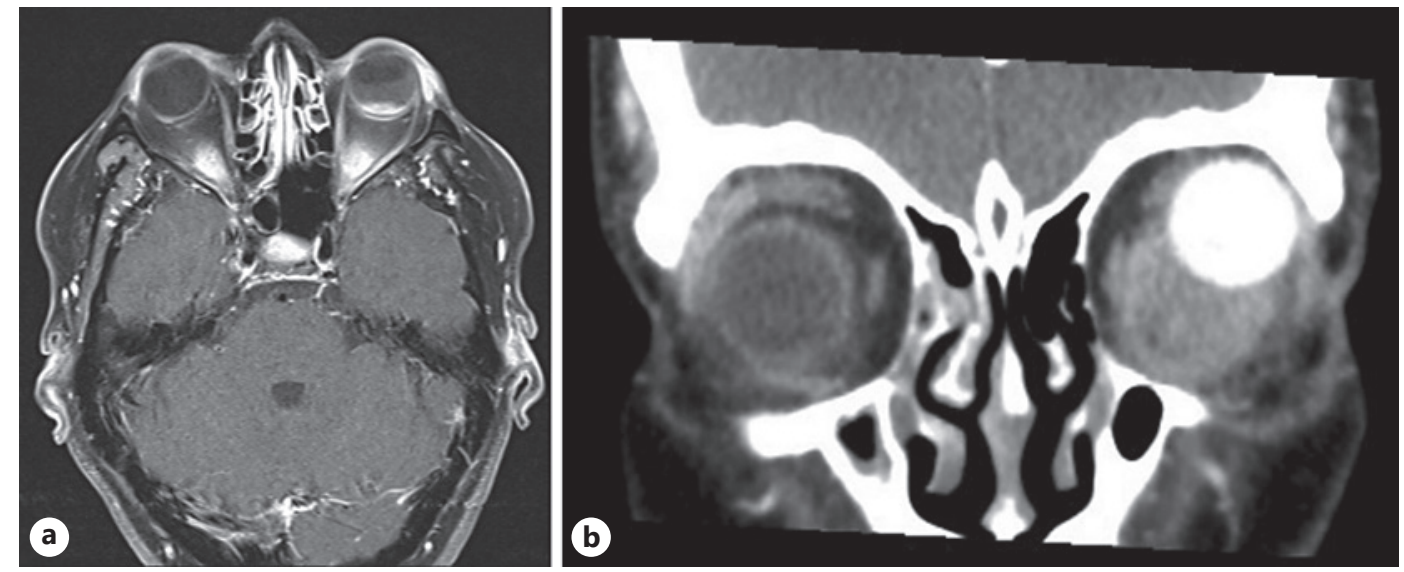

Fig. 1. MRI of the orbits on presentation and CT imaging 3 months after enucleation. a MRI orbits (axial scan, postcontrast, T1 with fat suppression) demonstrate an enhancing mass along the posterior left globe with associated total retinal detachment and an area of posterior scleral enhancement but no gross orbital disease. b CT orbits (coronal scan) after enucleation demonstrate a left heterogeneous inferomedial mass displacing the silicone implant superolaterally.
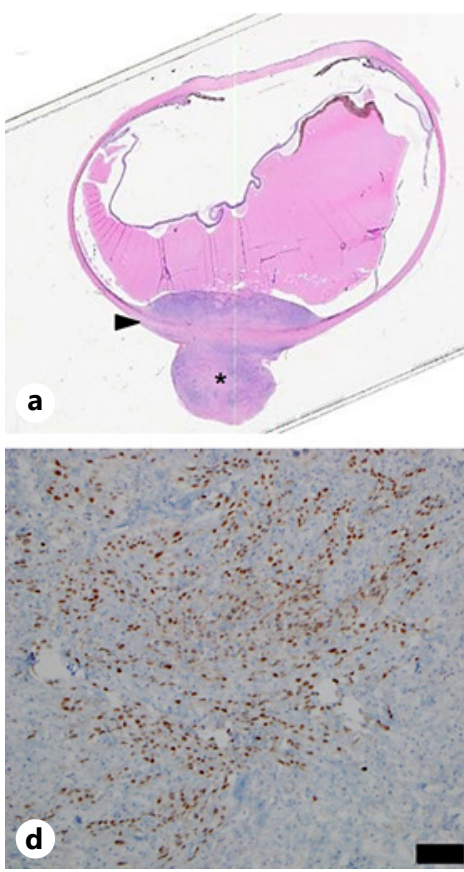
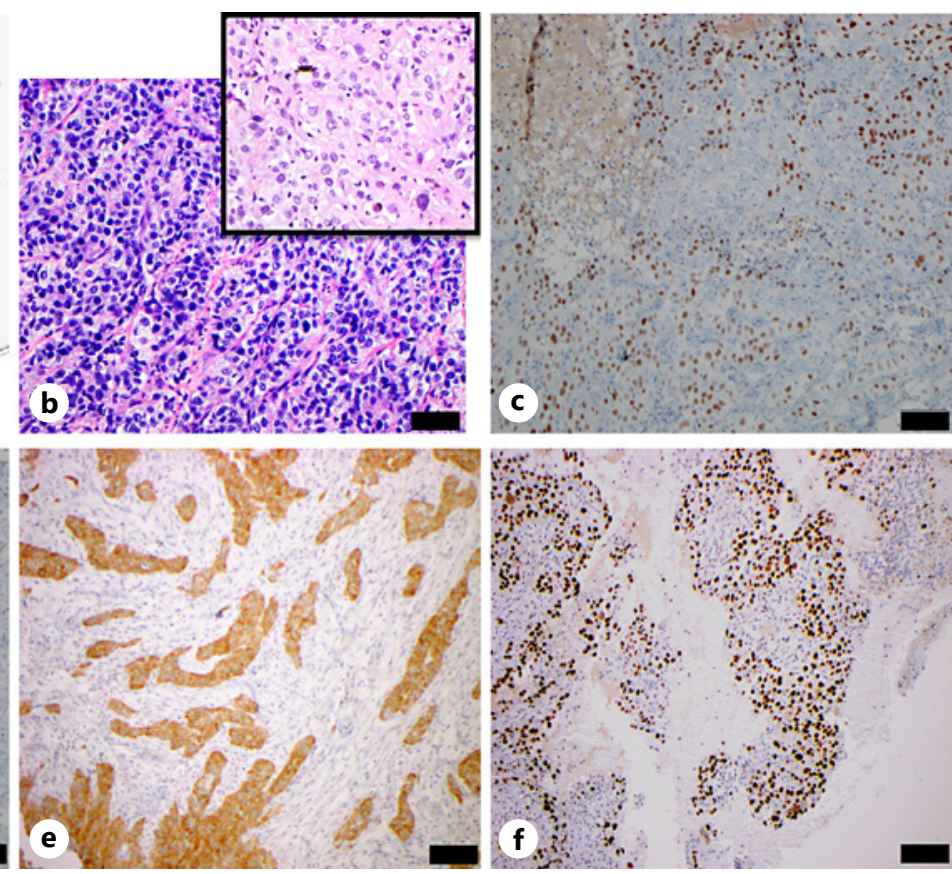

Fig. 2. a Left globe depicting a dome-shaped choroidal lesion (arrowhead) with an episcleral nodule (asterisk) (H\&E, panoramic view). b Nests of neoplastic cells with marked cytologic atypia and high mitotic activity infiltrating orbital soft tissue (H\&E. $\times 10)$. Inset: neoplastic epithelioid cells with eosinophilic vacuolated cytoplasm involving the choroid (H\&E. ×40). c-f Tumor cells are immunoreactive for TTF-1 (c), PAX-8 (d), BRAF (e), and p53 (f) ( $\times 10)$. Black scale bar, $500 \mu \mathrm{m}$.

some bizarre, giant cells with focal rhabdoid features involved the choroid (Fig. 2b). Eight to 10 mitoses per 10 high power field were identified. The neoplastic cells percolated the adjacent sclera and episcleral surface. Immunohistochemical (IHC) studies were performed using monoclonal antibodies encompassing the following: thyroid transcription factor 1 
(TTF-1), thyroglobulin, paired box protein 8 (Pax-8), and Ki-67 proliferative index. The malignant epithelioid cells were positive for TTF-1 and PAX-8, while negative for thyroglobulin (Fig. 2c, d). The Ki-67 proliferative index was high (>50\%). The combined histopathologic and IHC findings were consistent with ATC metastatic to the choroid with extraocular extension likely directly through adjacent sclera. Considering known, yet indolent, brain metastases, the multidisciplinary team elected to manage expectantly.

Three-months postoperatively, the patient complained of worsening left periorbital edema, erythema, and discomfort. She also reported that her ocular prosthesis looked "larger" and did not fit as well. A computed tomography scan demonstrated an enhancing mass in the left orbit (Fig. 1b). An orbital biopsy was performed for a high suspicion for malignancy, either by direct spread from the prior ocular lesion or, less likely, a discrete orbital metastasis. On histopathology, the tumor exhibited features similar to those previously described in the enucleation specimen. Additional IHC studies were ordered, including p53, B-type RAF kinase V600E (BRAF), and cytokeratin cocktail AE1/AE3 stains. The tumor cells were positive for BRAF, AE1/AE3, and p53 (>70\% of tumor cells) (Fig. 2e, f). The final histopathologic assessment confirmed metastatic ATC to the orbit. The positive staining for BRAF suggested that this tumor could represent a dedifferentiation of a preexisting well-differentiated thyroid carcinoma.

Understanding that further orbital surgery would be noncurative given metastatic disease elsewhere, the patient elected to proceed with exenteration to mitigate pain and reduce local tumor burden. The other known metastases to the brain were thought to be indolent, and her multidisciplinary oncology team determined that 5-year prognosis was good if the orbital disease was resected. Histopathological examination of the exenteration specimen was consistent with prior biopsies and again showed thyroid carcinoma. At 32 months since diagnosis and 12 months from exenteration, there was no evidence of orbital recurrence of disease. Her brain lesions appear stable on the most recent MRI.

\section{Discussion/Conclusion}

This report demonstrates an ATC that arose in association with a PTC. The primary tumor metastasized to lymph nodes of the neck retaining the papillary morphology while the undifferentiated anaplastic carcinoma was noted in the distant metastases to the eye and orbit.

The reasons for anaplastic behavior in the eye and orbit are not clear but could be induced by local microenvironment effects, development of angiogenesis, and stroma-tumor interactions, among other mechanisms. Since a prior thyroid malignancy is the greatest risk factor for ATC [7], we surmise the ATC represents a dedifferentiation of PTC. We did not pursue next-generation sequencing; however, cytomorphologic findings and IHC studies support these findings.

Consistent with known aggressiveness of ATC, the primary tumor demonstrated rapid growth, causing tracheal and vocal cord impairment manifesting as hoarseness, dyspnea, and stridor. Initial systemic imaging suggested no metastasis to bone or other distant sites. Despite initial radical surgical resection of the primary tumor with neck dissection, chemotherapy in combination with a PD-1 inhibitor [8], and high-dose radiation, the choroidal metastasis was symptomatic within 8 months of the initial diagnosis. At that time, EBRT was selected for the best balance of efficacy and morbidity to preserve vision. Other options included radioactive iodine ablative therapy or plaque brachyradiotherapy. Enucleation was considered, but preservation of vision and globe was deemed possible at that time. The subsequent refractory neovascular glaucoma and NLP vision made enucleation more

\section{Karger'}


clearly indicated. Interestingly, her presumed brain metastases responded much more favorably to SRS than the choroidal tumor did to EBRT; both sites were $<4 \mathrm{~mm}$ and treated within 1 week of discovery on neuroimaging. Due to surgical morbidity, no brain biopsy was performed; hence, these brain lesions remain presumptive thyroid carcinoma metastases that may or may not match the histologically proven ocular and orbital disease.

Recurrence in the orbit, whether from direct extension or metastasis, reinforces the need for close monitoring of patients with ATC. Many of the classic signs of orbital tumors, such as motility restriction or proptosis, may be less obvious in an anophthalmic socket or hidden underneath an ocular prosthesis. It is possible the episcleral nodule could have seeded the orbit directly, given posterior scleral enhancement seen on pre-enucleation MRI (Fig. 1a) and subsequent discovery during pathologic sectioning, or the orbit could have been seeded during the enucleation itself. However, there were no gross abnormalities of the globe or the orbit, and it was not until pathologic sectioning did the episcleral nodule became evident. Second, the time course of 3 months for symptom development favors a second metastasis over a direct extension. The possibility of ATC behaving this aggressively does necessitate the consideration of primary exenteration if ATC is known.

PTC is the most common thyroid carcinoma metastasizing to the orbit [9]. Metastatic ATC to the globe and orbit is extremely rare [7]. Anaplastic carcinoma can arise de novo or in association with a known PTC. The morphological spectrum of ATC includes 3 main histological patterns: spindle cell, giant cell, and squamoid. The latter is predominantly seen in our case. Immunohistochemically, ATC is generally negative for thyroglobulin and calcitonin while positive for pan-cytokeratin and epithelial membrane antigen in one-half and one-third of cases, respectively. PAX-8 has been reported positive in 79\% of all subtypes of ATC, although in $92 \%$ of ATC with squamoid pattern. TTF- 1 is generally negative, but a small percentage can be positive, particularly those with squamoid pattern [10]. Further, ATC with associated PTC has shown to be strongly positive for BRAF V600E by IHC, which correlates with DNA mutational analysis in prior studies [11]. It is noteworthy that this patient was also diagnosed with a serous borderline tumor of the ovary, which is associated with BRAF mutation in $35 \%$ of cases [12]. Additionally, mutational inactivation of TP53 with widespread nuclear overexpression of p53 has been reported in $70-80 \%$ of ATC.

Gupta et al. [6] reported the only other case of ATC with orbital metastasis. Their patient presented with ocular symptoms that led to diagnosis of the primary malignancy [6]. The present case behaved much more aggressively, and ocular involvement manifested after primary tumor resection. Distant metastases may be present in $50 \%$ of newly diagnosed ATC and another $25 \%$ will develop metastasis during the disease course [5]. Timely surgical treatment of orbital metastasis can be life extending in some scenarios [6]. Enucleation and orbital debulking alleviated pain and offered local tumor reduction in this patient. The impact of these surgical interventions on survival remains unknown, but this patient has remained stable from a systemic standpoint for at least 12 months after exenteration.

\section{Statement of Ethics}

This case report adhered to the ethical standards laid out by the Declaration of Helsinki. No identifying patient information, images, or likeness has been presented. Written informed consent was obtained from the patient for participation in this study and publication of the details of the medical case and any accompanying images. A signed consent form was completed by the patient in the presence of her nuclear family members, 3 of the co-authors, and our clinic staff. The approval of the protocol detailed in this case report was not required by the UC Irvine Institutional Review Board.

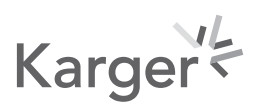


Tran et al.: Ocular and Orbital Metastasis from Thyroid Carcinoma

\section{Conflict of Interest Statement}

The authors acknowledge an unrestricted grant from Research to Prevent Blindness to the Gavin Herbert Eye Institute at the University of California, Irvine, and J.P.T., consultant, Horizon Therapeutics.

\section{Funding Sources}

No funding sources were used or involved in any way in this case report.

\section{Author Contributions}

S.C., T.T., M.D.V.E., and J.T. wrote the manuscript and were directly involved in the patient's care. S.C. and T.T. performed the detailed review of the patient's clinical course and the literature review.

\section{Data Availability Statement}

This is a case report with protected health information that is protected by HIPAA.

\section{References}

1 Shields JA, Shields CL, Scartozzi R. Survey of 1264 patients with orbital tumors and simulating lesions: the 2002 montgomery lecture, part 1. Ophthalmology. 2004 May;111(5):997-1008.

2 Ohtsuka K, Hashimoto M, Suzuki Y. A review of 244 orbital tumors in Japanese patients during a 21-year period: origins and locations. Jpn J Ophthalmol. 2005 Jan-Feb;49(1):49-55.

3 Bonavolonta G, Strianese D, Grassi P, Comune C, Tranfa F, Uccello G, et al. An analysis of 2,480 space-occupying lesions of the orbit from 1976 to 2011. Ophthalmic Plast Reconstr Surg. 2013 Mar-Apr;29(2):79-86.

4 Besic N, Luznik Z. Choroidal and orbital metastases from thyroid cancer. Thyroid. 2013 May;23(5):543-51.

5 Molinaro E, Romei C, Biagini A, Sabini E, Agate L, Mazzeo S, et al. Anaplastic thyroid carcinoma: from clinicopathology to genetics and advanced therapies. Nat Rev Endocrinol. 2017 Nov;13(11):644-60.

6 Gupta D, Chappell M, Tailor TD, Keene CD, Moe K, Jian-Amadi A, et al. Orbital metastasis of undifferentiated/ anaplastic thyroid carcinoma. Ophthalmic Plast Reconstr Surg. 2015 Sep-Oct;31(5):e120-3.

7 Smallridge RC, Ain KB, Asa SL, Bible KC, Brierley JD, Burman KD, et al. American Thyroid Association guidelines for management of patients with anaplastic thyroid cancer. Thyroid. 2012 Nov;22(11):1104-39.

8 Bastman JJ, Serracino HS, Zhu Y, Koenig MR, Mateescu V, Sams SB, et al. Tumor-infiltrating T cells and the PD-1 checkpoint pathway in advanced differentiated and anaplastic thyroid cancer. J Clin Endocrinol Metab. 2016 Jul;101(7):2863-73.

9 Haugen BR, Alexander EK, Bible KC, Doherty GM, Mandel SJ, Nikiforov YE, et al. American Thyroid Association management guidelines for adult patients with thyroid nodules and differentiated thyroid cancer: the American Thyroid Association Guidelines Task Force on thyroid nodules and differentiated thyroid cancer. Thyroid. 2016 Jan;26(1):1-133.

10 Ragazzi M, Ciarrocchi A, Sancisi V, Gandolfi G, Bisagni A, Piana S. Update on anaplastic thyroid carcinoma: morphological, molecular, and genetic features of the most aggressive thyroid cancer. Int J Endocrinol. 2014; 2014:790834.

11 Ghossein RA, Katabi N, Fagin JA. Immunohistochemical detection of mutated BRAF V600E supports the clonal origin of BRAF-induced thyroid cancers along the spectrum of disease progression. J Clin Endocrinol Metab. 2013 Aug; 98(8):E1414-21.

12 Grisham RN, Iyer G, Garg K, Delair D, Hyman DM, Zhou Q, et al. BRAF mutation is associated with early stage disease and improved outcome in patients with low-grade serous ovarian cancer. Cancer. 2013 Feb 1;119(3): 548-54. 